\title{
La judicialización del femicidio en el centro del Ecuador: estudio comparativo de las provincias de Cotopaxi y Tungurahua
}

\author{
Mathías Valdez - Duffau ${ }^{1}$ Jessica Guashca -Vega ${ }^{2}$
}

Fecha de recepción: 17 de Noviembre 2017

Fecha de aceptación: 11 de Diciembre 2017

\section{Resumen}

El presente artículo se cuestiona cuál es el estado de situación de la judicialización de los casos de femicidio. Para ello desarrolla una investigación de campo en los distritos judiciales de dos provincias de la sierra ecuatoriana: Tungurahua y Cotopaxi. De ese modo, se logra evidenciar la evolución, en el periodo 2014-2017, de dichas investigaciones.

Palabras clave: Femicidio, Violencia, Justicia Penal.

\section{Abstract}

This article questions what is the state of the situation of the judicialization of cases of femicide. For this, a field investigation in the judicial districts of the provinces of the Ecuadorian highlands: Tungurahua and Cotopaxi, was conducted. In this way, it was possible to demonstrate the evolution, in the period 2014-2017, of said investigations.

Keywords: Femicide, Violence, Criminal Justice.

\section{Introducción}

Las cifras evidencian una situación de gravedad en relación con la violencia de género y a sus consecuencias. Según datos del Plan Nacional de Erradicación de la Violencia (2010), en Ecuador 6 de cada 10 mujeres han vivido algún tipo de violencia de género siendo Morona Santiago, Tungurahua y Pichincha las provincias que mayores índices de violencia de género. A la vez, 9 de cada 10 mujeres divorciadas en Ecuador han vivido una de las cuatro formas de violencia de género: psicológica, física, sexual o patrimonial. Según datos del Instituto Nacional de Estadística y Censos (INEC, 2014) la tasa de muertes de mujeres es de 0,72 por cada 100 mil habitantes. En Tungurahua 9 de cada 10 mujeres ha vivido algún tipo de violencia de género. Asimismo, el $91 \%$ de las mujeres ha sufrido violencia física en sus relaciones de pareja frente al $87,3 \%$ del total nacional.

En la primera parte del 2017, desde el 1 de enero al 31 de mayo, y tan sólo en el servicio de emergencias ECU-911 de Ambato, se registraron 3.082 llamadas que reportaron algún caso de violencia intrafamiliar. Según Montesdeoca (2017), director zonal encargado de la central de emergencias, de un total de 3.082 llamadas por violencia antes mencionado, unas 2.158 , casi un $70 \%$ por ciento, son situaciones de inminente peligro, en las que existe un grado de violencia alto y entre una pareja. Con relación a la judicialización de la violencia, cabe mencionar que, si bien en la Fiscalía de Ambato no se registran las estadísticas de homicidios desagregados según el sexo de la víctima ni por la agravante de femicidio, y de acuerdo con fuentes judiciales (Arauz 2016) se pudo establecer que los casos de asesinatos cometidos a mujeres son, en la mayoría de los casos, caratulados como femicidios.

Conforme a Rodas (2005) el femicidio es considerado un crimen de odio contra las mujeres. Así materializa el punto culminante de una espiral de violencia originada en la relación desigual con el sexo femenino. Dicha violencia femicida se entiende como un resultado de prácticas sociales, culturales, judiciales y políticas que avalan el comportamiento agresivo de los varones. Asimismo, Cabo y Maldonado (2005) examinan al femicidio desde la órbita criminal al entenderlo como un delito que se efectúa contra el género femenino a través de diferentes tipos de violencia, sea física, sexual, psicológica o patrimonial, la cual es propiciada por un autor del género masculino de modo de ejercer dominación sobre la mujer. Para Trujillo (2011) existen factores socioculturales que se articulan desde el entramado familiar debido a que:

"La madre enseña a sus hijas, que las mujeres tienen que ser hacendo- sas, fieles, piadosas. Se olvidan de ciertas capacidades intelectuales, atributos nuevos de las características de ser mujer, de su fortaleza sicológica, capacidad intelectual, cualidades que se consideraban exclusivas de los hombres" (pp. 30).

En materia legal, la normativa ecuatoriana es clara en cuanto a la protección de la integridad física y la vida. La Carta Magna establece en su artículo 66 (Cons. 2008, art 66) que garantiza el derecho a la vida, a la integridad física, psíquica, moral y sexual, así como el derecho a disfrutar de una vida libre de violencia en el ámbito público y privado. En el plano supranacional el Ecuador ha ratificado la Convención Interamericana para prevenir, sancionar y erradicar la violencia contra la mujer (CIPSEVM 1994), también conocida como "Convención de Belem do Para" la cual garantiza el derecho de toda mujer a una vida libre de cualquier forma de violencia, tanto en el ámbito público como privado. Además, proclama el "derecho de la mujer a ser valorada y educada libre de comportamientos y prácticas sociales y culturales basadas en la inferioridad" (art. 2).

En materia penal, el Código Organico Integral Penal (COIP, 2013) tipifica en su artículo 141 el delito de femicidio cuando "La persona que dé muerte a una mujer por el hecho de serlo o por su condición de género" y taxativamente establece cuatro agravantes al tipo penal básico. Ellos son (COIP, 2013, art. 142):

"Circunstancias agravantes del femicidio. Cuando vaya una o más de las siguientes circunstancias:

1. Haber pretendido establecer o restablecer una relación de pareja o de intimidad con la víctima.

2. Exista o haya existido entre el sujeto activo y la víctima relaciones familiares, conyugales, convivencia, intimidad, noviazgo, amistad, compañerismo, laborales, escolares o cualquier otra que implique confianza, subordinación o superioridad.

3. Si el delito se comete en presencia de hijas, hijos, o cualquier otro familiar de la víctima.

4. El cuerpo de la víctima sea expuesto o arrojado en un lugar público."

\section{Metodología}

¿Cuál es el estado de situación judicial del femicidio a partir de su tipificación en el Código Orgánico Integral Penal en la provincia de Tungurahua en comparación con la provincia de Cotopaxi en el periodo 2014-2017?

El presente artículo halla su justificación e importancia en el análisis de la faz de respuesta judicial a un espectro de casos particulares de violencia que aquejan a la sociedad ecuatoriana. La manifestación de violencia de género con consecuencia de la pérdida de vida, refleja esquemas de conducta de una sociedad en donde los conflictos se resuelven de forma violenta, la misma que se debe a relaciones desiguales del poder de las mujeres en la sociedad. En el aspecto filosófico la investigación se valió de un paradigma crítico propositivo debido a que busca la solución de un problema por medio de la investigación, partiendo de las causas del mismo y planteando la solución que se le dará por medio de una investigación aplicable y favorable para la colectividad.

\section{Resultados}

Mediante esta investigación en base al tema del femicidio en el periodo 2014-2017 se llegó a obtener los siguientes resultados, que se han logrado alcanzar mediante las entrevistas realizadas a las distintas unidades de investigación. Las mismas que han proporcionado información verídica que permite resolver la pregunta de investigación. A

PhD en International Public Policy por la Universidad de Osaka (Japón). Docente-Investigador de la Dirección de Investigación y Desarrollo de la Facultad de Jurisprudencia y Ciencias Sociales de la Universidad Técnica de Ambato.UTA. Ambato-Ecuador.Email: me.valdez@uta.edu.ec

2Estudiante-Investigadora de la Carrera de Derecho de la Facultad de Jurisprudencia y Ciencias Sociales de la Universidad Técnica de Ambato. UTA. Ambato- Ecuador. Email: jessicaguashca@gmail.com 
través de la entrevista realizada en la unidad de investigación de la Fiscalía Provincial de Tungurahua, se pudo obtener los datos estadísticos del delito de femicidio en el periodo 2014-2017 mediante el cual se lo representa a través de un gráfico de pastel en el cual podemos visualizar que se ha dado un total de 202 casos, mientras tanto que la sentencia que se ha dictado a las personas que han cometido este delito ha sido de 53 casos que representa un 26\%, en cuanto a la instrucción fiscal se ha dado un total de 85 con un porcentaje del $42 \%$ y por último los casos que se encuentran en investigación son un total de 64 con un porcentaje de $32 \%$.

Tabla 1. Estadística de femicidio - Tungurahua 2014-2017

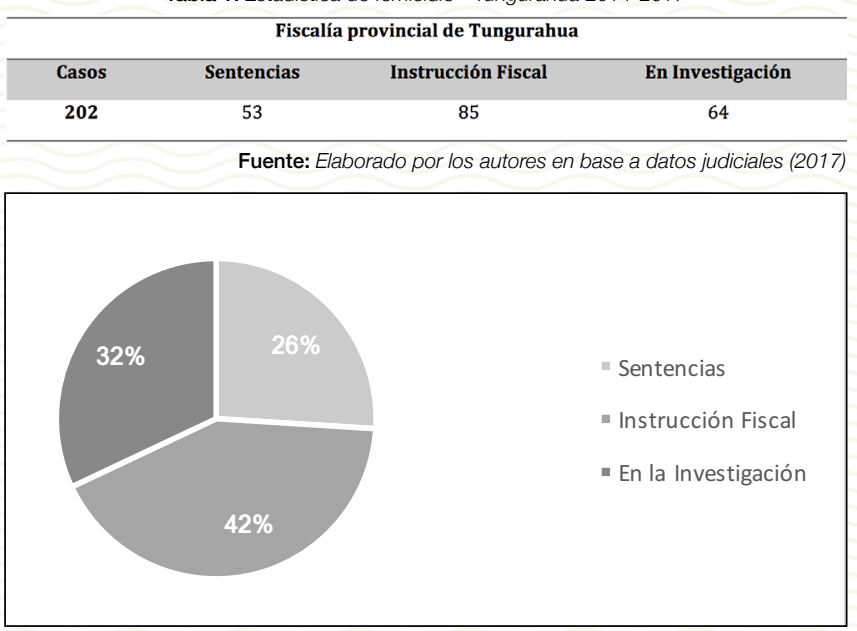

Fuente: Elaborado por los autores en base a datos judiciales (2017) Casos tramitados por Fiscalía periodo 2014-2017 Figura 1. Estadística de femicidio Tungurahua 2014-2017

Mediante el diagrama de pastel se puede identificar que en la unidad de investigación de la DINASED en el periodo 2014-2017 se ha dado un total de 178 casos de femicidio dando así 82 sentencias respecto a este delito con un pequeño porcentaje del $46 \%$ siendo todas estas causas de femicidio con un total de 96 casos dando así un porcentaje final del $54 \%$.

Tabla 2. Estadísticas de femicidio Tungurahua 2014-2017 (DINASED)

\section{DINASED}

\begin{tabular}{ccc}
\hline Casos & Sentencias & Casos de Femicidio \\
$\mathbf{1 7 8}$ & 82 & 96 \\
\hline
\end{tabular}

Fuente: Elaborado por los autores en base a DINASED (2017)

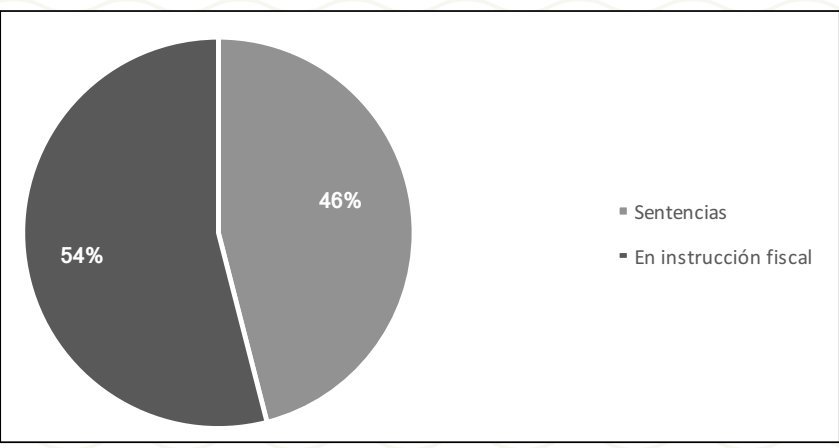

Fuente: Elaborado por los autores en base a datos de la DINASED (2017) Casos reportados por DINASED periodo 2014-2017

Figura 2. Estadística de "femicidio" Tungurahua 2014-2017 según DINASED

De acuerdo con la información proporcionada por parte de las Unidades de Policía Comunitaria (UPC) se pudo evidenciar que en el periodo 2014-2015 se dio un total de 4.000 llamadas de auxilio, de las llamadas receptadas, entre ellas 255 llamadas, reportaban casos de femicidio con un porcentaje del $7 \%$, mientras que 1.055 llamadas fueron para reportar violencia intrafamiliar con el $26 \%$. Mientras tanto 885 llamadas fueron receptadas por casos de abuso sexual con un porcentaje no mínimo del $22 \%$, en cuanto a violencia física se reportaron 955 llamadas con un porcentaje del 24\%, y por último se dio un número de llamadas un poco más alto a los anteriores con 850 llamadas con respecto a maltrato psicológico dando así un total de $21 \%$, una vez dado a conocer los números de llamadas se ha podido determinar el número de casos de femicidio.

Tabla 3. Estadísticas de femicidio Cotopaxi 2014-2017

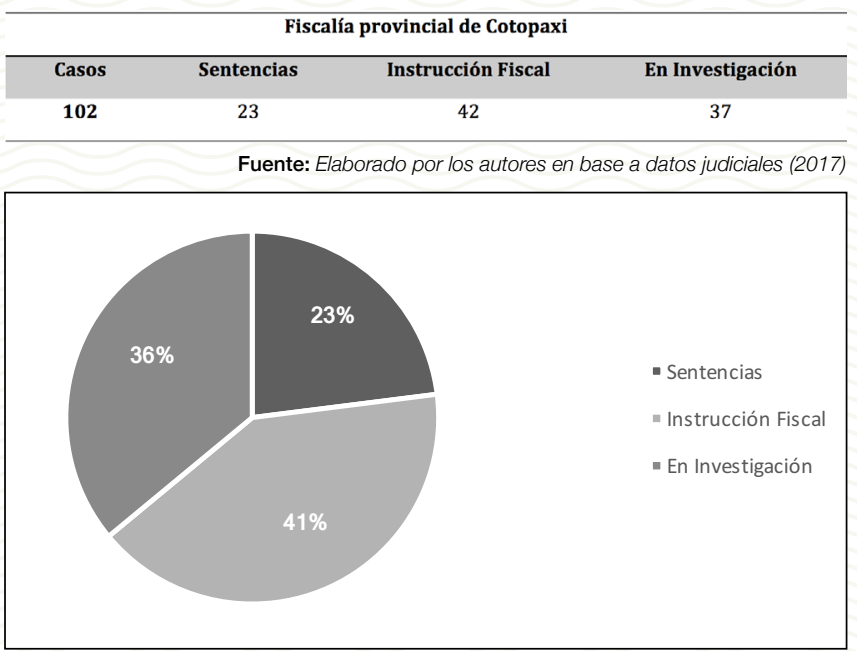

Fuente: Elaborado por los autores en base a datos judiciales (2017)

Figura 3. Estadísticas de casos de femicidio Cotopaxi en trámite por Fiscalía 2014-2017

Como se puede visualizar a través de los datos obtenidos de la unidad de investigación de la DINASED arroja como resultado que en el periodo 2014-2017 se ha dado un total de 71 casos, de los casos producidos el $39 \%$ representa a sentencias y el $61 \%$ son casos de femicidio.

Tabla 4. Estadísticas de casos femicidio Cotopaxi 2014-2017 (DINASED)

\section{DINASED}

\begin{tabular}{ccc}
\hline Casos & Sentencias & Femicidio \\
\hline $\mathbf{7 1}$ & 28 & 43 \\
\hline
\end{tabular}

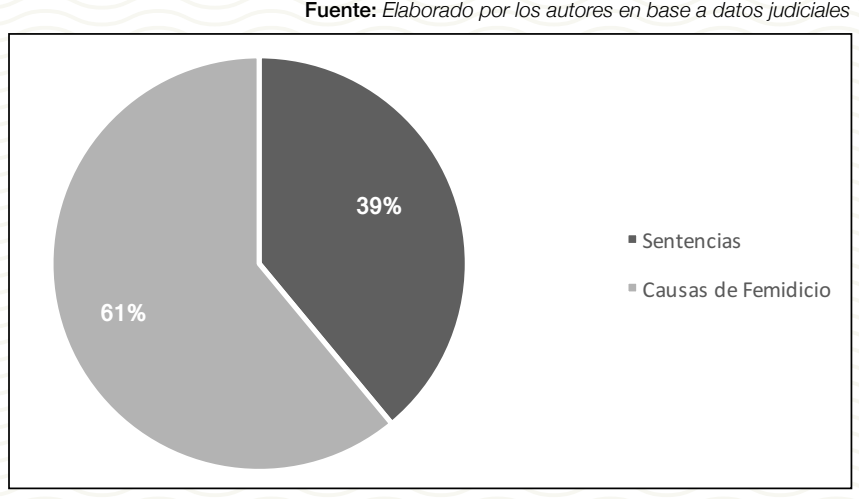

Fuente: Elaborado por los autores en base a datos judiciales Figura 4. Estadísticas de casos femicidio Cotopaxi 2014-2017 (DINASED)

La entrevista realizada a las Unidades de Policía Comunitaria revela que se ha registrado un total de 1.500 llamadas de auxilio, las cuales representan el $50 \%$ del total, mientras que 50 llamadas son de casos de femicidio con el $2 \%$ de las llamadas receptadas, 550 son casos de violencia intrafamiliar con el $18 \%$, en cuanto a abuso sexual un total de 350 llamadas con 12\%, violencia física 300 llamadas resultando así 10\%, 250 son llamadas de maltrato psicológico siendo el 8\% del total de llamadas receptadas. 
Tabla 5. Estadística de casos de femicidio Cotopaxi 2014-2017 (UPC)

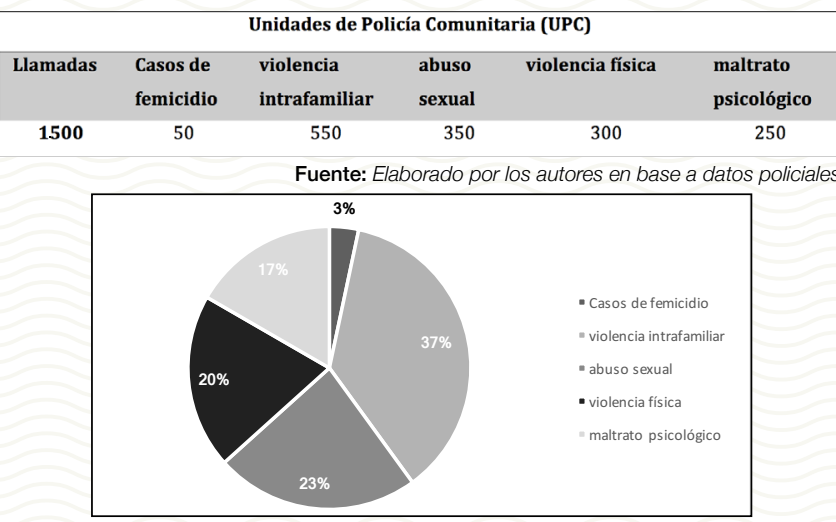

Fuente: Elaborado por los autores en base a datos policiales Figura 5. Estadística de casos de femicidio Cotopaxi 2014-2017 (UPC) A través de los datos receptados de las distintas unidades de investigación de las dos provincias se puede distinguir que en la provincia de Tungurahua es donde existe un mayor índice de casos de femicidio, un total de 202 en el periodo analizado, en comparación con Cotopaxi que registra 102, es decir, la mitad. Teniendo en cuenta que Tungurahua actualmente cuenta con una cifra poblacional de 504.583 de los cuales 259.800 son mujeres y 244.783 son hombres, en cuanto a Cotopaxi la población total es de 409.205 tanto que la población de mujeres es de 210.580 y dando así la cantidad de 198.625 que representan hombres, la tasa de femicidio es de 1 caso cada 1.287 mujeres en Tungurahua, mientras que en Cotopaxi es de 1 caso por cada 2.058 mujeres de dicha provincia.

Una vez visualizada la diferencia poblacional entre la provincia de Tungurahua y la de Cotopaxi, además de los casos de femicidio producidos, se establece que Tungurahua es la provincia con mayor índice de femicidio, dejando en evidencia que en Cotopaxi existe una tasa menor de casos de femicidio, pero con igual número de sentencias hacia los casos que cometen este delito.

Tabla 6. Comparativo de Datos estadísticos femicidio periodo 2014-2017

\begin{tabular}{|c|c|c|c|c|}
\hline & & & Tungurahua & Cotopaxi \\
\hline \multirow{13}{*}{ 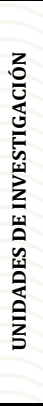 } & \multirow{4}{*}{ Fiscalía provincial } & Casos & 202 & 102 \\
\hline & & Sentencias & 53 & 23 \\
\hline & & Instrucción fiscal & 85 & 42 \\
\hline & & En investigación & 64 & 37 \\
\hline & \multirow{3}{*}{ DINASED } & Casos & 178 & 71 \\
\hline & & Sentencias & 82 & 28 \\
\hline & & Femicidios & 96 & 43 \\
\hline & \multirow{6}{*}{$\begin{array}{l}\text { Unidades de Policía } \\
\text { Comunitaria }\end{array}$} & Llamadas & 4.000 & 1.500 \\
\hline & & Casos & 255 & 50 \\
\hline & & Violencia intrafamiliar & 1.055 & 550 \\
\hline & & Abuso sexual & 885 & 350 \\
\hline & & Violencia física & 955 & 300 \\
\hline & & Maltrato psicológico & 850 & 250 \\
\hline
\end{tabular}

Fuente: Elaborado por los autores en base a datos judiciales (2017)

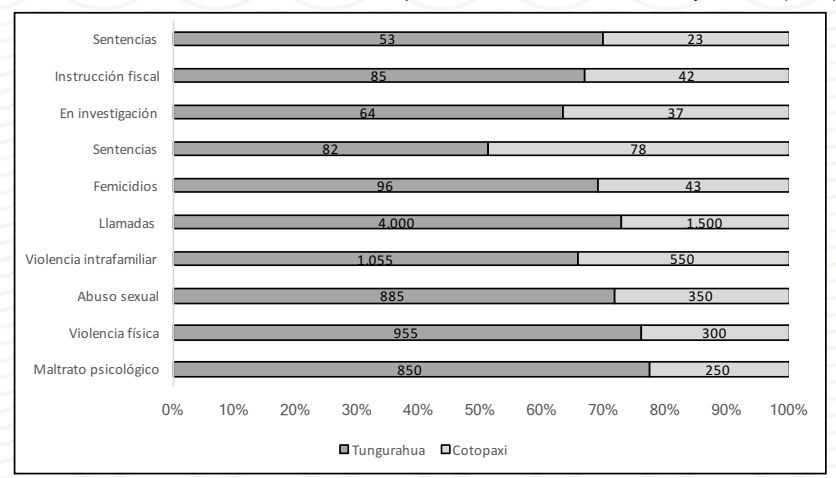

Fuente: Elaborado por los autores en base a datos judiciales (2017) Figura 6. Cuadro comparativo de Femicidio en Tungurahua y Cotopaxi periodo 2014-2017

\section{Conclusiones}

La presente investigación se aboca a responder el siguiente interrogante: ¿Cuál es el estado de situación judicial del femicidio a partir de su tipificación en el Código Orgánico Integral Penal en la provincia de Tungurahua en comparación con la provincia de Cotopaxi en el periodo 2014-2017? La investigación evidencia que, en la provincia de Tungurahua, existe un elevado índice de casos de femicidio cuando se lo compara con Cotopaxi. Ello se evidencia en que, en el periodo analizado, en la provincia de Tungurahua existieron 202 casos de femicidio de los cuales el $26 \%$ de los casos cuentan con una sentencia condenatoria, mientras que en la provincia de Cotopaxi existen 102 casos de femicidio de los cuales el 23\% cuentan con una sentencia condenatoria. De lo expuesto se desprende dos cuestiones. La primera que en la provincia de Tungurahua el índice de femicidio en comparación con la provincia de Cotopaxi es del 61,48\%. Es decir, que existe más del doble de casos para el mismo periodo. La segunda cuestión es que, no obstante, la tasa de sentencias condenatorias es muy similar en ambos distritos, uno de cada cuatro casos, debido a que el $25 \%$ de los casos por femicidio, en cuanto a la relación casos/condenas, obtienen una sentencia condenatoria.

A modo de recomendaciones vale mencionar que en materia jurídica se debería modificar el Art.142.-del Código Orgánico Integral Penal ya que no se encuentra tipificado como agravante de femicidio que el hecho se lo cometa bajo los efectos del alcohol o sustancias sujetas a fiscalización. En materia de política pública, se recomienda que se faculte atribuciones a los Tenientes Políticos y Comisarios Nacionales de Policía con el fin que se conceda medidas de protección a las víctimas sobrevivientes de tentativa de casos de femicidios. En relación con la sociedad civil, se recomienda intensificar, a nivel local y provincial, campañas de concientización para la sociedad en general y para las mujeres en particular acerca de la violencia y de modos alternativos de resolución de conflictos.

\section{Referencias}

Aldana, M. (5 de julio de 2017). Femicidio. (J. Guashca, Entrevistador)

Amoros, C., \& Alvarez, A. d. (2005). Introducción: Teoría feminista y movimientos feministas. Madrid: Miverva Ediciones.

Andina, U. (21 de julio de 2010). Informe sobre los derechos humanos Ecuador. págs. 1-5.

Ávila, R., Salgado, J., \& Valladares, L. (2009). El Género en el Derecho. Quito: V\&M Graficas. vistador)

Bonifaz, P. (4 de julio de 2017). Femicidio. (J. Guashca, Entre-

Caputi, J., \& Rusell, D. (1990). El femicidio sirve de modelo a otras formas de violencia. Lamarea.com, 1-2.

Código Orgánico Integral Penal. (2014).

Constitución de la República del Ecuador. (2008).

Convención Interamericana de Derechos Humanos. (1978).

Hurtado, J. (2000). Femicidio criterio ideológicos y recurso al derecho penal. Navarra: Fibrourg.

Monarrez, J. (2000). La cultura del femicidio en ciudad Juárez.

23-25.

Entrevistador).

Montesdeoca, M. (5 de julio de 2017). Femicidio. (J. Guashca, trevistador)

Proaño, M. (12 de julio de 2017). Femicidio. (J. Guashca, Entrevistador)

Quispe, R. (28 de Julio de 2017). Femicidio. (J. Guashca, En-

Rodas, R. (2005). 100 años de femicidio en el Ecuador. Revista renovación, 25-30. trevistador)

Romero, R. (24 de junio de 2017). Femicidio. (J. Guashca, En-

Rusell, D. (2016). Femicidio una perspectiva Global. España: Harmes ediciones.

Sagot, M. (2000). Femicidio en Costa Rica. San José, Costa Rica: colección teórica $N^{\circ} 1$.

Sánchez, J. (2014). La evolución de los derechos de la mujer. eHow en español, 4-11.

Yépez, M. (2014). El femicidio en el COIP. Revista Judicial, 3-6. 